\title{
THE NATURE OF THE OPTICAL EMISSION IN RADIO-SELECTED AGN.
}

\author{
M. T. WHITING \\ University of New South Wales \\ E-mail: mwhiting@phys.unsw.edu.au \\ R. L. WEBSTER, P. MAJEWSKI, A. OSHLACK \\ University of Melbourne \\ P. J. FRANCIS \\ Australian National University
}

\section{Modelling radio-loud AGN in the optical}

We investigate the optical emission from radio-loud AGN using the Parkes Half-Jansky Flat-spectrum Sample $\left(\mathrm{PHFS}^{3}\right)$, a sample with a much broader range of quasar colours ${ }^{4}$ than typical optically-selected samples.

We use a comprehensive dataset ${ }^{4}$ of quasi-simultaneous BVRIJHK photometry to model the optical-near-infrared emission. A simple power law fit is compared to a "disc+jet" model with: i) a blue power law representing accretion disc emission; and ii) a synchrotron component with an exponential turnover. Details of the fits can be found elsewhere ${ }^{7}$. About $40 \%$ of the stellar sources show evidence for synchrotron emission, explaining well the bulk of the colour distribution.

A similar number of sources show no evidence for synchrotron, and are dominated by the accretion disc. They are thus similar to optically-selected quasars. Identification of the true strength of accretion disc emission is $\mathrm{vital}^{8}$ for accurate modelling of multi-wavelength spectra, particularly when considering the high-energy emission and its inverse-Compton origin.

The reddest sources appear to be dominated by strong dust absorption rather than synchrotron. The presence of dust should be confirmed by observations of thermal emission by the dust or absorption by the associated molecular gas. 


\section{Synchrotron turnovers and an AGN's optical appearance}

The synchrotron component turns over sharply at some critical frequency, due to an upper limit in the electron energy distribution within the jet. The location of this turnover is a fitted parameter, and a large range of values is seen, spanning all observed frequencies. Additionally, since the blue quasars (i.e. no optical synchrotron) are known to have synchrotron dominating the radio emission (they are compact, flat-spectrum sources), their synchrotron component must turn over somewhere in the infrared. This proposition should be testable with ground- and space-based infrared observations (a similar effect has been seen with ISO in the $200 \mathrm{mJy}$ sample $^{1}$ ).

If such a synchrotron component were to exist in a BL Lac object, the BL Lac would be mis-identified. The lack of significant accretion disc emission will leave starlight from the host galaxy to dominate the optical. The BL Lac will thus appear as a flat-spectrum radio galaxy. This is similar to the effect discussed by Marchã \& Browne ${ }^{5}$, but occurs due to the energetics of the jet, rather than the overall luminosity.

\section{Radio flux limit and the AGN population}

It has been found ${ }^{2,6}$ that the lower the radio flux limit of a flat-spectrum sample, the greater the fraction of sources that appear optically as passive elliptical galaxies, rather than obvious AGN (eg. quasars). While the overall AGN luminosity will be an important factor in this effect, the synchrotron turnover should also play a part, through a decrease in turnover frequency with decreasing radio flux. We can relate this in a broad sense to the energetics of the jet: powerful jets, with bright radio emission, have many high-energy particles that radiate at high frequencies (i.e. in the optical), while less powerful, fainter jets lack significant numbers of such particles. This study highlights the need for consideration of jet properties in modelling the flat-spectrum source population.

\section{References}

1. S. Antón, Ap\&SS 285, 257 (2003)

2. A. Caccianiga et al., MNRAS 329, 877 (2002)

3. M. Drinkwater et al., MNRAS 284, 85 (1997)

4. P. Francis, M. Whiting \& R. Webster, PASA 17, 56 (2000)

5. M. Marchã \& I. Browne, MNRAS 275, 951 (1995)

6. J. Muñoz et al., ApJ 594, 684 (2003)

7. M. Whiting, R. Webster \& P. Francis, MNRAS 323, 718 (2001)

8. M. Whiting, P. Majewski \& R. Webster, PASA, 20, 196 (2003) 